\title{
The Grand Challenges: A Potential Boost for a Responsible Research and Innovation
}

\author{
Giovanni Colombo \\ Research Center, Istituto Superiore Mario Boella (ISMB), Italy
}

Copyright (C) 2015 by authors, all rights reserved. Authors agree that this article remains permanently open access under the terms of the Creative Commons Attribution License 4.0 International License

\begin{abstract}
The need to adopt an ethical attitude in Research and Innovation (R\&I) is growing with the increasing power of scientific and technological developments and the huge impact they can create on society and environment. However, the effects of research and innovation are difficult to predict and this makes quite hard to inspire a priori the action of researchers and innovators towards credible objectives of social benefit. The paper puts forward some new arguments about the feasibility of a responsible research and innovation approach applied to the grand challenges, namely the strategic issues of universal relevance like food and water safety, sustainable mobility, global warming. It is shown how grand challenges can foster a responsible attitude in R\&I due to their specific nature and the level of social involvement they demand. Key enablers of the responsible approach are the integration of social disciplines since the design of the R\&I Projects and the association of renewed education models to the R\&I initiatives conceived according to responsibility criteria. Two examples, taken from the innovation topics arising respectively in sustainable energy and heating sectors support the abstract considerations.
\end{abstract}

Keywords Responsible Research and Innovation, Innovation Policy, Anticipatory Approach, Grand Challenges, Interdisciplinary Approach, Education Models

\section{Introduction}

According to common sense, responsibility is the spirit that should inform the action of any rational person towards his neighbour. Throughout all mankind history, the term responsibility has been associated to a number of different cases. Scientific and technological development has significantly enlarged the variety of circumstances where the principle of responsibility is called into question. Research and Innovation (R\&I) are the key development factors and the boosters of the ever growing and globalised process of goods production and consumption. So, the effects of any
R\&I action, the associated responsibility and the significance of neighbour may become extremely wide in both space and time dimensions. As a result, a profound dilemma is emerging about R\&I initiatives: they may at the same time guarantee the wellbeing of our society and threaten the sustainability of the planet, hence the life conditions on earth.

The concept of responsibility has been extensively challenged in the last century. In the late seventies, Hans Jonas has produced a valuable philosophical construction in relation to the huge power of technology and the imperative for advanced societies to be aware of and accountable for the consequences that an irresponsible use of technology can produce [1].

Today more than ever, human communities are facing the urgent need to transform social and economic processes in order to ensure at the same time environmental sustainability and social prosperity. The scientific community must be conscious of the fundamental role it can play in such a global challenge and humble enough to recognise that all dimensions of society have to become essential actors of these transformations.

The European Union is looking at the R\&I function under a more and more comprehensive horizon. Societal and environmental objectives are now complementing knowledge creation and competitiveness aims of the Community and this is visible in the European policies and R\&I supporting measures. Furthermore, in the last decade the Union is devoting a considerable attention to the issue of Responsible Research and Innovation (RRI) [2] and the responsibility criterion is deeply embedded in the Horizon 2020 Programme, where smartness, cohesion and sustainability values are viewed as essential components of the economic and social development. Along this line, responsibility in R\&I should adopt and pursue values like: social engagement, creative learning, accountability in the choices. And the society should be able to govern the evolutionary processes according to these principles [3]. Quite similar institutional attention is manifested in all the industrialised areas, see for instance [4], [5] and is growing significantly in the developing countries. The issue of how to 
translate the above values in concrete measures has been extensively studied and for instance, in [6] the assessment of some alternative R\&I policies and their potential impacts has raised the need to re-enforce the responsibility character of the Community through suitable policy measures. On the same wave, the ethical considerations have been put in relation to regulatory and policy aspects and the values of a balance between socio-cultural diversity and the share of benefits through an inspired governance of scientific advances have been asserted [7].

An amazing number of scholar papers and books have supported these fundamental positions and have created a valuable background of new concepts, classifications and models around the responsibility in research and innovation. The following conjectures are constructed over this basis and the relevant references are recalled along the discourse. This paper addresses, with a reflective spirit, two central aspects of responsibility in R\&I, in the framework of Grand Challenges (GCs), namely: the expectation building of a given social aim and the mechanisms through which such an expectation can be anticipated through RRI actions. Section 2 deals with the source, the motivations and the methodological approach. Section 3 elaborates on the reasons why the GCs represent the easiest way to consolidate important aspects of responsibility in R\&I and section 4 shows the plausibility of this conjecture by considering a couple of innovation cases in energy and health sectors. The concluding remarks are reported in Section 5.

\section{Methodological Aspects}

The principles of responsibility have been explored by the Author on the basis of a long experience in the field of Information and Communication Technologies (ICT) and the consciousness of the growing role that these techniques are acquiring in the transformations of society-sensitive processes. The generic nature of ICTs is confirmed not only by the many sectors they can be applied to, but also by the range of different strategic perspectives that can be associated to their use. Staying at this strategic dimension, the potential of ICTs can be exploited either to rationalise [8] existing processes (e.g. digitalise a booking service) or, much more radically, to define completely new processes according to longer term visions. The latter case singles out a stronger intentionality and may generate significant impacts, up to affecting the cognitive mechanisms of individuals or the collective behaviour of a community. The so called sharing economy has grown on this constitutive character of the ICTs and has indubitably produced profound effects on modern society.

But the intriguing circumstance is that these technologies, thanks to the virtualisation, modelling and networking functions they are able to expose, can contribute significantly to the construction of future perspectives and the evaluation of the joined innovation initiatives. These remarks on the relevance of ICT-based transformations and the abstraction power of ICT, represent for many ICT experts a natural way to view through different glasses the issues raised by the Grand Challenges and the ethical values that are called into question [9]. The GCs offer in this way a unique opportunity to gather under the same dilemma (RRI principles, policy and governance) forces coming from different experiences.

The dual character of the ICTs is quite useful to tackle from a methodological point of view the following issues about responsibility. The intentionality of a potential innovation may in fact shape the character of a RRI.

In this exercise, it is apparent that whenever a technology advance is used to understand the nature of the matter (e.g. sub-atomic laws) or the essence of a process (e.g. the stability conditions of a micro climate) the responsibility for such an action cannot be substantially questioned. Here in fact, the intentionality of the technology-supported research is to understand the phenomenon and possibly to bridle its behaviour in a formal representation. The relevant research action is far from offering some application evidence; hence, not only the claim to predict its consequences is unrealistic, but applying a rigid scrutiny to the action may threaten the research freedom and autonomy and raises the risk of narrowing its potential benefits. In this case, a responsible attitude should foster a public dialogue around the research object and a prompt contamination at the level of higher education, where the new issues and discoveries could be viewed on the watermark of the consolidated disciplines.

Conversely, whenever the technology is used to manipulate either the natural matter or pre-existing processes, the social implications of the innovation action and the social consequences become more evident. And the need arises to choose the appropriate action according to ethical criteria. If the innovation action is more responsive to a responsibility-driven scrutiny, at the same time it is significantly dragged by market and business opportunities which are very seldom ethically inspired. In any case, this kind of innovation discontinuity is the one where a RRI-based policy should be more strongly pursued. Such a policy should address awareness, engagement and collective responsibility by fostering a diffuse understanding of the needs the innovation is expected to fulfil and a transparent evaluation of the potential impacts through the integration of scientific, economic and social cultures.

The innovation initiatives tied to the Grand Challenges are indubitably manipulations of pre-existing processes or new solutions for the emerging social or economic needs. In this sense they deserve a renewed attention in the perspective of RRI.

\section{Responsible Research and Innovation in Grand Challenges}

As stated by the European Programme Horizon 2020 [10] the GCs address the variety of issues emerging along the society's development path. Dealing with problems like 
global warming, health and demographic changes, energy sustainability, water shortage, waste production and disposal cycles, mobility of humans and goods, requires radical changes of the relevant systems and processes. At both Union's and Region's level, the issues generated by GCs are faced in public research programs that in principle offer a concrete financial and cooperative base to develop a RRI.

Looking at the European research framework, the paper puts forward some new remarks and proposals about the principles of a RRI discussed in basic articles by Stilgoe et al. [11] and Pandza, et al. [12] where the authors have developed the anticipatory approach as a way to face the responsibility issue which is also considered in recent EU documents [6].

The anticipation is an attempt to bypass the need to predict the social impact of a R\&I action. In the discourse on responsibility, the prediction is expected to support the a priori scrutiny of the action-generated effects. However, it is universally recognised that such a claim is quite problematic, due to the number of unpredictable variables that may affect the outcome of the innovative action. Not only the prediction is a difficult exercise, but the a priori evaluation of its accuracy and confidence degree is almost impossible because of the time that inevitably elapses from the innovation design phase to the point when its effects can be measured. Moreover, the innovation action is generally modifying a complex process, subject per se to random conditioning factors and this makes the impact, if possible, even more unpredictable.

The anticipatory approach, proposed by Stilgoe, et al. [11] and introduced in a more general form by Rosen [13], is supported by the existence of common and transparent objectives reflecting the social expectation about the issue tackled by the innovation action. As requested by the participatory character of democracy, all the social dynamics (scientific and social research, business, politics) should participate to the design of such expectation, as stated in Leydesdorf [14]. The anticipatory approach requires that all the agents involved in the innovation instance assume a collective responsibility about the innovation choice.

In a way, this means embedding the unfeasibility of prediction into the collective character of responsibility. If considered in conjunction with the expectation that inspires backwards the innovation choices, this approach means shaping a desirable future (anticipation) through a responsible innovation rather than trying to predict the effects of a loosely directed innovation act. The prediction of the innovation effects resembles more a projection, carried out to evaluate to what extent the effects are moving towards the expectation.

In the light of this background, the two key issues of the paper, namely the expectation building and the implementation of the anticipatory approach can be discussed.

In the framework of GCs, the expectation is a dynamic representation of urban systems in a long-term horizon. The representation is backward inspiring the present choices and in the opposite direction it is absorbing the consolidated signals of the technological, economic and social progress as well as the lessons learned by the previous experiences (knowing the past is the only way to measure the effect of any action). The necessary participation of the Public Administration [15] is a guarantee that the expectation is more than an abstract description of aspirations, and it is rather offering a trace for anticipate their achievement in the social interests [16].

Building the expectation in the framework of GCs is also a way to connect directly the deliberation act to the aims of the research action, and to challenge the consistency of political choices in terms of innovation outcomes to be consolidated (exploitation) and the way adopted to do so (model). This may substantially help in overcoming the present limits of politics in deliberation and on deliberation considered in [17]. The transformations induced by GCs are experienced locally and locally re-defined according to the social acceptance. It is in the local dimension (city, village, rural community) that the culture is nurtured also taking into account the new challenges. The local dimension is where the expectation can be built with the highest degree of social awareness and this is an additional element suggesting to use GCs as the terrain where responsibility can grow.

When tackled through an anticipatory approach, GCs can create favourable conditions to grow social involvement and reflexivity and they can significantly contribute to consolidate new models of knowledge creation and education.

The transformations to be anticipated under the umbrella of a collectively defined expectation are naturally favoured by the social character of the innovation driven by GCs. But there is a stronger element fostering a responsible attitude through an anticipatory approach in GCs: the circumstance that the needed transformations would simply not be possible without a new level of social involvement (engagement, responsiveness) and a profound identification with the common aims. This very pragmatic need is conveying the transformation efforts in the same direction addressed by a responsible R\&I: an unexpected reinforcement of the relevant policies.

Economy and Sociology of GCs reflect the anticipatory model: the innovation issues of today are inspired by the expectation and, at the same time, the innovative changes create new knowledge (coded and tacit knowledge) and new behaviours which contribute in turn to the continuous update of the expectation. This is mirroring the definition of reflexivity [18]. In fact, in this context, the circular interaction between the innovation issues and the current innovative changes through the expectation, leads the issues themselves and the R\&I design to depend on the innovation outcomes. Reflexivity tends to be a natural characteristic of any innovation process driven by GCs.

Grand Challenges raise research issues and speculative processes that are not (yet) framed into consolidated disciplines [18]. This kind of research is in fact so much dependent on the context in which the social transformations 
are expected to take place that elements of tacit knowledge cannot be disregarded in the design and execution of the investigation process. In addition, this research is trans-disciplinary in a double sense. On the one hand it requires that scientific, economic, social and humanistic disciplines share a common responsibility in setting-up and conducting the innovative initiatives, on the other hand, the success and the value of the action depends much more on the ability to orchestrate the various actors (effectiveness of the ensemble) rather than on the effectiveness of any singular discipline. The latter concept is familiar in mathematical studies of complex systems and non-linearity; in economic studies concerns the composite production chains; in the field of innovation models reflects the open and user innovation models. The limited space the publishing context offers to this kind of new speculations compared to the width of magazine, journals and conferences offered to the canonical disciplines cannot be an obstacle in the process of fostering the creation of this kind of knowledge at both research and education levels.

The education in particular should deserve greater attention within the responsibility framework in Grand Challenges. The key choice in this respect is to associate higher education courses embedding the responsibility values to the R\&I programs dealing with GCs. These courses should be radically re-directed according to the emerging knowledge-creation mechanisms and draw attention to responsibility tangles. This sort of cognitive congruence is a catalyst of responsibility along the social transformations.

Both the reference to a shared expectation and the anticipatory approach prefigure a continuous presence of social forces and social disciplines in the R\&I process. In GCs, this may foster the public and informed discourse about generic technologies like e.g. ICT and nanotechnologies [17]. In fact, the elaboration of potential solutions for GCs issues, confines to a comprehensible subset of cases the field of potentially countless applications these technologies can give rise to, and gives a preliminary substance to the potential effects of these applications.

Summarising, it is through the sense of engagement and responsiveness that the community builds-up its future and plays an active role in the development process. So, the R\&I issues generated by the GCs, can help answering the basic question raised by a responsible approach : why do we want to realise this innovation and how? And this in alternative to the more conventional argument: let's try to predict where the actual trends will bring us in order to be ready to adopt the most suitable innovations.

\section{Applications and Examples}

A couple of examples may help to understand the unique opportunity the Grand Challenges represent on the path towards a RRI. The examples address two sectors undergoing radical, R\&I-based transformations with a significant impact on society: sustainable energy and health

\section{processes.}

In the last few decades, the growing environmental issues have brought the industrialised countries to foster the development of renewable energy sources by adopting new regulations and economic incentives. The growing density of renewable energy sources is now substantiating the move of the energy paradigm from centralised solutions, where the energy is produced by high capacity generators and distributed as far as the final user, to decentralised systems where some renewable energy (from sun, wind, biomass) is produced, transformed, exchanged and used locally.

The energy community, the configuration that best suits the emerging paradigm, is becoming feasible thanks to new norms and technological advances on energy production and storage [19]. An energy community is a set of energy users/producers adopting a common solution to fulfil their energy requirements by gaining economic and environmental benefits. The community principles, namely resource sharing and integration of needs, produce a surplus of value compared to the total value achieved through individual configurations.

From a pure technical view, the dilemma to be resolved in order to maximise the community benefit is how to deal with two random (and basically unpredictable) functions: the energy demand and the energy production the community exhibits in any instant (see Figure 1). Unfortunately, these two functions do not fit: the demand is produced by the unaware community members acting as energy consumers in that instant and the renewable energy is produced by sources (e.g. sun, wind), obeying natural laws which are on turn unaware of the community's needs of energy. Some actions can lead the two curves to fit: importing (buying) or exporting (selling) energy to the network; storing energy for a subsequent use (when energy is produced in excess); using energy previously stored for the community needs. In this framework, adopting the real time control actions able to optimise the community benefit is quite a difficult task and its solution can be faced through a significant R\&I effort by a suitable assortment of energy engineers, mathematicians and information technology experts.

But the intriguing circumstance is that the problem can be radically shuffled if the behaviour of energy consumers is considered (as it should be) as variable of the overall question. The level of engagement and responsiveness of the community members make them available to either adopt a more frugal use of energy or accept a time shift of the energy they intend to use. This further variable of the optimisation problem requires that the social sensibility and the tools to encourage it are taken into account since the community design phase and continuously monitored or tuned along the community operations. Hence, the community members need to be aware in advance of the aims of the coming innovation (expectation); their active role represents a necessary condition for the collective success of the innovation discontinuity and in addition, the solution requires that a strong multidisciplinary approach is put in place. The energy community is a small scale, though a complete proof of how 
the anticipatory approach can inspire the social transformations in the framework of grand challenges.

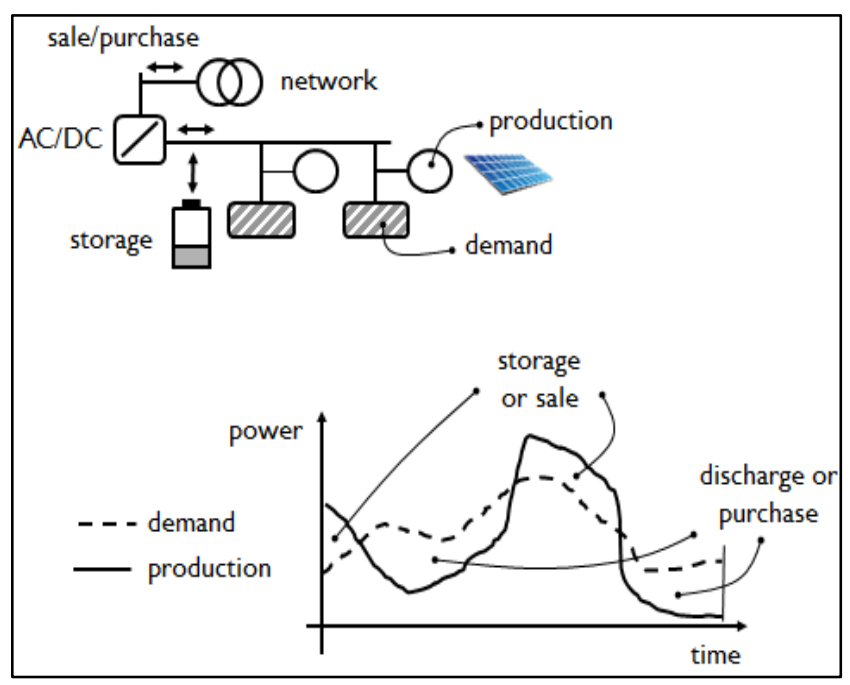

Figure 1. Energy community, configuration and demand-production curves

The importance of literacy and involvement in energy saving has been demonstrated in a wide number of experimentations and in some cases these attitudes resulted in a truly renewed users' behaviour. Many experimental programs proved that the behavioural factors in energy savings may account up to $50 \%$ of the energy reduction [20]. In all the cases, the level of awareness of the environmental issues, namely a future-oriented culture has been a key success factor, a point of social attraction and a guarantee for the stabilisation of the new practises [21]. A recurring characteristic of such experiments is the time-varying relationship between the influencing factors and the behavioural and energy consumption practises. Accordingly, education policies and collective conventions (social norms) must be reactive to these dynamics [22].

The savings obtained through community-based initiatives are quite promising and may reach figures of $20 \%$ reduction of carbon emissions. In many cases the most significant savings have been achieved through a pure informative (non-technical) intervention. A comprehensive scrutiny of the importance of a collective approach to the energy saving issues is given in [23] where a sensitivity analysis is performed about the "Low Carbon Communities". Key elements for the effectiveness and the economic survival of these Communities are: the adoption of strategies for a wide systemic change (expectation), the Community's engagement (awareness) and the learning activities associated to Community creation plans and operations. These elements are also reflected in the emerging policy proposals, focalised on normative, economic and political perspectives [19].

Some personal and technical factors may foster a responsible orientation of the R\&I Projects. Personal factors are the environmental culture, values and social sensibility of the user/producer. Among the technical elements, a timely feed-back (real time metering) about the energy saved thanks to a behavioural discontinuity may be essential [24]. The effectiveness of such a feed-back is heavily depending on the level of reflexivity and consciousness the innovation act has been able to grow with the involved users.

The effects of direct and indirect feedback to the user may produce energy savings ranging from $5 \%$ to $25 \%$ fully ascribed to the behaviour changes [25].

The health processes are quickly moving from an illness-based to a prevention and rehabilitation-based approach. This statement is far from being an empty keyword because it induces a number of radical transformations of the health systems. The practice of prevention does not apply uniquely to healthy people, it concerns also high-risk categories and may minimise the relapse probability for people who overcome the acute phase of a disease (second level prevention). Similar healthy approaches are based on reinforcement of abilities and rehabilitation cares in case of post-traumatic events and cognitive diseases like autism and senile dementia. Moreover, the progress of clinical and pharmaceutical care and the application of ICT-based mechanisms, are widening the proportion of population suffering from chronic diseases who, in spite of this, can conduct a normal life by adopting an healthy life style and performing directly some basic care functions possibly controlled from a remote medical site.

These de-hospitalised forms of health care aim at improving wellbeing and widen the period of active life in the ageing perspective. They may also lighten the cost of public care and assistance that would otherwise become rapidly unsustainable due to demographic changes and the significant growth of elder population [26].

The above scenario is feasible thanks to the medical and technological advances, but an effective decentralised and personalised care strongly requires the patient to develop consciousness, literacy and self-management capabilities. So, the innovation of non-acute health processes does require that the health system users and care givers, up to the level of family components and assistance professionals are deeply engaged in the definition of the new procedures and responsive to their tuning needs along the operative phases.

There is a strong evidence of the advantages that consciousness, self-management and literacy can produce on wellbeing. A comprehensive analysis of the incidence of life styles, and self-management on the health conditions for chronic diseases can be found in [27]. The self-management approach is particularly effective in chronic diseases, due to the multiplicity of causes, the unclear role of technology and the time-varying behaviour of chronicity compared to the cases of acute diseases [28]. A self-management approach is productive provided that it is practised within an interdisciplinary process, including psychological, biological and social interventions, and all this may work just in presence of individual ability, behavioural and emotional responses of the subject [29]. The same paper underlines the importance of contextual conditions for a full exploitation of self-management potentials (for instance, individual and 
group-based approaches may achieve different results and imply different levels of economic sustainability).

The benefits induced by an informed self-management approach range from an improved status in well-being (including the remission of depression and distress), self-efficacy, control of symptoms, patients' knowledge. Moreover self-management reduces the pace of decline and the risk of relapses after an acute phase [30].

In any case, in order to produce durable benefits, these practises must enter permanently in the behavioural habits of the patient. For instance the diabetes self-management education can produce significant effects in the care of type 2 diabetes, just after the completion of an education phase, and the efficacy of this behavioural intervention decreases if the education is not renewed at a sufficient pace [31]. Symmetrically, a patient-centred care approach has to be adopted from the clinical side and framed within suitable public health protocols [32].

Again, a responsible approach of the health-related R\&I action should be constructed since the beginning on the multiplicity of disciplines and sensitivities concurring to a higher degree of wellbeing (from psychology to social science to clinical practise to engineering).

\section{Discussion and Concluding Remarks}

The two innovation examples on sustainable energy and health processes demonstrate how the anticipatory approach can encourage a responsible attitude in research and innovation actions whose success is strictly tied to the social involvement and consciousness.

Whenever the innovation chances of a socially-sensitive process are just assigned to the enabling capability of a new technology, the risk of failures is quite significant. For instance, if the problem of energy saving at domestic level is seen as a pure question of technological improvements of plants and insulation, the investment cost may often exceed the economic advantages induced by the gained efficiency as Fowlie et. al. have recently pointed out [33]. This is also due to a rebound effect, which is leading the final energy users to a relaxed attention to the energy consumption in presence of some $a$ priori and non-participatory guarantee offered by the purely technical renewal of plants. On the other hand, it is universally recognised that GCs are prefiguring a new kind of society, growing on the basis of a common perspective. Now, due to its systemic character, the transition cannot rely only on the growing technological power and its applications, but also on the new social models the democratic process will be able to distil. So, concepts like personal awareness, cultural growth, social cohesion, personal and collective liability should become key factors of the innovation dilemma and all the relevant disciplines have a fundamental role in that.

In this way, we are moving from a standpoint where social involvement is considered to be a necessary factor for implementing technical innovation actions to a standpoint where social issues are native constituents of the innovative conjecture.

The above considerations put forward the idea that grand challenges can create favourable conditions to experiment and consolidate a responsible attitude starting from the phase when the direction of the innovation action is established. It is apparent that any innovation facing a grand challenge is naturally looking at a background of potentially negative circumstances the action is expected to prevent (e.g. global warming, drop of welfare, corruption of the environment). It can be said that these circumstances will occur with a high level of confidence if current processes and behavioural habits are not discontinued. The knowledge of the negative circumstances and their likelihood support the need for an action and offer some criteria to choose ex-ante the most appropriate action among the many possible.

The EU research strategies and the Regional plans based on structural funds are considering the social benefit among the objectives to be achieved by the candidate projects and frequently require that social bodies and social disciplines are involved. Indubitably these requirements encourage a responsible attitude of the participating partners, but they are still too feeble signals for the affirmation of a responsible R\&I. In the EU and Regional projects dealing with the grand challenges, a more responsive attitude could be fostered through some practical governance rules.

Firstly, social bodies and social disciplines should be allocated from the very beginning of the project and should play a central role in identifying the project's objectives and the approaches to be adopted to achieve them. Too often, the innovation is fully driven by a technological or process advance and the social variables are just invoked as second order elements, by neglecting to consider them as primary research objectives. A more active role of social bodies and disciplines would assign each actor a greater responsibility about the effects of the innovation step and the inevitable innovation-tuning initiatives.

Each partner of the project should be aware of the perspective driving the innovation action and should identify its work as a contribution to a collective aim. This attitude requires that the project governance is able to stress the benefits that a collective effort can generate for all the parties in case the project is successful. The leverage lies in the completeness of the innovation effects, which is guaranteed when the project requires that new models are explored in the business, social behaviour and educational dimensions.

The above cases can support a RRI approach just if the project is well formed in terms of complementarity of partners, shared intentionality and clear guarantees of continuity and consolidation in case of success. The pre-commercial procurement of innovation fulfils (only) some of these requirements.

Finally, it is important to recognise that higher and professional education can become an acceleration and anticipatory factor for RRI. To achieve this, the education forces (academia, research centres) taking part in an innovation program should allow that the new speculative issues the project is tackling permeate and possibly re-direct 
the pedagogic schemes they are usually conforming to. In this way, a well-formed and well-governed project conveys at the education level the responsibility character and the consciousness of the coming innovation when it is still in the pre-market phase. The association between R\&I and the education programs has been fruitfully experimented in the Programs of the European Institute of Innovation and Technology (EIT) and its Knowledge and Innovation Communities, acting on the European scale [34], [35]. There are even stronger reasons to adopt the same approach in the regional dimension where the innovation of socially-sensitive processes and the local education policies are (should be) part of the same public plan.

Summarising, the grand challenges seem to offer interesting hints to face the RRI dilemma. In fact, the innovation projects they are urging embed societal needs in a natural way and stimulate the adoption of a collective responsibility through an anticipatory approach. A lot of theoretical and experimental work is still needed to fully exploit this potential in the framework of the common perspective created through the democratic process and the cultural advances of society.

\section{Acknowledgements}

The Author is very grateful to Edoardo Calia and Giancarlo Pirani for having shared their expertise in discussing and challenging the paper's conjectures and to Giuseppina Fausto for the essential support spent in the paper preparation.

\section{REFERENCES}

[1] Jonas H.. The Imperative of Responsibility: In Search of Ethics for the Technological Age', University of Chicago Press.

[2] De Saille S.. Innovating innovation policy: the emergence of "Responsible Research and Innovation"', Journal of Responsible Innovation Vol. 2 n. 2.

[3] European Commission. Responsible Research and Innovation - Europe's ability to respond to societal challenges. : ; 2012. https://ec.europa.eu/research/swafs/pdf/pub_rri/KI0214595E NC.pdf.

[4] Ethics Education in Science and Engineering. National Science Foundation 2010.

[5] Cultivating Cultures for Ethical Science, Technology, Engineering and Mathematics. National Science Foundation 2014.

[6] European Commission. Options for strengthening Responsible Research and Innovation; 2013. https://ec.europa.eu/research/science-society/document libra ry/pdf_06/options-for-strengthening_en.pdf.

[7] Directorate General for Research and Innovation . Ethical and regulatory challenges to science and research policy at the global level. 2012.

http://ec.europa.eu/research/science-society/document librar y/pdf_06/ethical-and-regulatory-challenges-042012_en.pdf.

[8] Colombo G., Pio Magnani N., Minerva G., Scarrone E.. Network engineering issues in the UMTS development perspective. Journal of Communications and Networks 2000; N. 2(N. 1): .

[9] Colombo G., Ferrero F., Pirani G., Vesco A.. Planning Local Energy Communities to Develop Low Carbon Urban and Suburban Areas, IEEE International Energy Conference.

[10] European Commission, Horizon 2020. Workprogramme 2014-2015 Science with and for Society; 2013. https://ec.europa.eu/research/participants/data/ref/h2020/wp/ 2014_2015/main/h2020-wp1415-swfs_en.pdf.

[11] Stilgoe J., Owen R., Macnaghten P.. Developing a framework for responsible innovation. Elsevier - Research policy November 2013; N. 42(N. 9) .

http://www.sciencedirect.com/science/article/pii/S00487333 13000930.

[12] Pandza K., Ellwood P.. Strategic and ethical foundations for responsible innovation. Elsevier - Research policy June 2013; N. 42(N. 5) .

[13] Rosen R.. Anticipatory Systems: Philosophical, Mathematical and Methodological Foundations. Pergamon Press 1985.

[14] Leydesdorff L.. The Knowledge-based Economy: Modelled, Measured, Simulated. Universal Publishers Boca Raton 2006.

[15] European Commission. European Innovation Partnership on Smart Cities and Communities; 2013. http://ec.europa.eu/eip/smartcities/files/sip_final_en.pdf

[16] Shulz-Shaeffer I., Kubischok N., Meister M.. Visionary Technoscientific Practices: the Co-Constitution of Practice and Imagination, S. NET 6th annual meeting.

[17] van Oudheusden M.. Where are the politics in responsible innovation? European governance, technology assessment and beyond . Journal of Responsible Innovation 2014; N. 1(N. 1).

[18] Nowotny H., Scott P., Gibbson M. . 'Mode 2' Revisited: The New Production of Knowledge. Minerva 2003; N. 41.

[19] UK Department of Energy and Climate Change. Community energy strategy: full report. 2014.

https://www.gov.uk/government/uploads/system/uploads/att achment_data/file/275163/20140126Community_Energy_St rategy.p $\overline{\mathrm{df}}$

[20] Fisher J., Irvine, K.. Reducing household energy use and carbon emissions: the potential for promoting significant and durable changes through group participation', IESD PhD Conference - Energy and Sustainable Development Leichester, UK.

[21] Darby S.. Smart metering: what potential for householder engagement?, Building research \& information 38 (5).

[22] European Environment Agency. Achieving energy efficiency through behaviour change: what does it take?', EEA Technical report No 5. ISSN 1725-2237.

[23] Mayne R., Derby S., Hamilton J.. EVALOC Evaluating Low 
Carbon Communities - Individual and social influences on energy use - A literature review for the EVALOC Project', Oxford Brookes University.

[24] Shove E.. Converging conventions of comfort, cleanliness and convenience. Journal of Consumer Policy 2003; Vol. 26(N. 4) .

[25] Ehrhardt-Martinez K., Donnelly K., John A.. Advanced metering Initiatives and Residential Feedback Programs: A Meta-review for Household Electricity-Saving Opportunities; 2010.

http://www.energycollection.us/Energy-Metering/AdvancedMetering-Initiatives.pdf.

[26] European Commission. The 2015 Ageing Report- economic and budgetary projections for the $28 \mathrm{EU}$ Member State (2013-2060); 2013.

http://ec.europa.eu/economy_finance/publications/european economy/2015/pdf/ee3_en.pdf (accessed ).

[27] World Health Organisation. Global status report on non-communicable diseases; 2014.

http://www.who.int/global-coordination-mechanism/publicat ions/global-status-report-ncds-2014-eng.pdf?ua=1 .

[28] Holman H., Lorig K.. Patient self - management: a key to effectiveness and efficiency in care of chronic disease . Stanford University School of Medicine Public Health Report 2004; N. 119.

http://www.publichealthreports.org/issueopen.cfm?articleID $=1370$.
[29] Barlow J., Wright C., Sheasby J., Turner A., Hainsworth J.. Self-management approaches for people with chronic conditions: a review Patient education and counselling. ELSEVIER 2002; (48) .

[30] Lorig K., Sobel D., Ritter P., Laurent D., Hobbs M.. Effect of a self-management program on patients with chronic diseases. Effective Clinical Practise 2001 .

[31] Norris S., Lau J., Smith S., Smith C., Engelgau M.. Self-management education for adults with type 2 diabetes. Diabetes care $2002 ; 25(7)$.

[32] Robinson J., Callister L., Berry J., Dearing K.. Patient-centred care and adherence: definitions and applications to improve outcomes. Journal of the American Academy of Nurse Practitioner 2008; N. 20

[33] Fowlie M., Greenstone M., Wolfram C.. Do energy Efficiency Investments deliver? . NBER Working Paper 2015; N. 21331 .

[34] European Institute of Innovation \& Technology. Analysis of sinergies fostered by the EIT in the EU innovation landscape; 2013.

http://eit.europa.eu/sites/default/files/Analysis_of_Synergies. pdf .

[35] European Institute of Innovation \& Technology. Catalysing innovation in the knowledge triangle; 2012. $\mathrm{http}$ ://eit.europa.eu/sites/default/files/EIT_publication_Final. pdf. 